\title{
Road-Map für die elektronische Krankengeschichte für die Hausarztpraxis
}

\section{Heinz Bhend, Marco Zoller}

Arbeitsgruppe SGAM.Informatics

Korrespondenz:

Dr. med. Heinz Bhend

Oltnerstrasse 9

CH-4663 Aarburg

heinz.bhend@sgam.ch

\section{Hintergrund}

Wie schon verschiedentlich kommuniziert, hat die Schweiz bei der Nutzung von elektronischen Krankengeschichten in den Arztpraxen einen gewaltigen Nachholbedarf. Dieser Umstand kann deshalb zum Problem werden, weil damit die ambulanten Praxen vom Informationsfluss abgekoppelt werden, was wiederum ihre Rolle beeinflusst.

Neueste Erhebungen [1] zeigen, dass nur etwa $11 \%$ der niedergelassenen Ärzte vollständig auf elektronische Dokumentation umgestellt haben. Die weitaus brisantere Aussage dieser Studie ist, dass zwei Drittel der befragten Kollegen eine Umstellung in den nächsten drei Jahren ausdrücklich nicht erwägen. Ein wichtiger Faktor für dieses Zögern sind die fehlenden Standards für die elektronische Krankengeschichte, was Datenaustausch und Datenmigration behindert und einen Anbieterwechsel massiv erschwert. Mit der Lancierung einer Road-Map für elektronische Krankengeschichten möchten wir einen pragmatischen Weg einschlagen, um die Diskussion anzuregen und praktikable Lösungen zu skizzieren.

Da viele Kolleginnen und Kollegen überfordert sind mit der Frage, nach welchen Kriterien eine Softwarelösung zu beurteilen ist, möchten wir unsere Minimalvorstellungen für ein praxistaugliches System kommunizieren. Wenn ein Produkt diese Vorgaben im neutralen Test erfüllt, wird ihm das Label der Arbeitsgruppe SGAM.Informatics zuerkannt. Für die praktizierenden Ärzte stehen zudem Checklisten zur Evaluation der Produkte sowie Workshops zur Planung der Umstellung auf eine papierarme Praxis zur Verfügung.

\section{SGAM.Informatics-Label für elektronische $\mathrm{KG}$}

Das Ziel eines Labels «SGAM.Informatics» ist vor allem die Vereinheitlichung der Systeme auf der Basis eines Minimaldatensets. Dieses Vorgehen soll in Zukunft den Datenaustausch zwischen den verschiedenen Softwarelösungen ermöglichen.

Wir befassen uns bewusst nur mit dem Teil der klinischen Dokumentation und setzen voraus, dass die administrativen Daten bei Bedarf meist mehr oder weniger problemlos transferiert werden können. Zudem müssen Rechnungsdaten bei einem Systemwechsel nicht transportiert werden können.

\begin{abstract}
Beispiel
Herr Huber zieht um von A nach B. Der bisherige Hausarzt Dr. Müller hat in den letzten drei Jahren eine elektronische Krankengeschichte über Herrn Huber geführt. Nach Datum sind Notizen, Blutdruckmesswerte und Labordaten digital vorhanden. Auch die verschriebenen Medikamente, deren Wechsel, genaue Dosierung und Unverträglichkeiten sind dokumentiert. Dr. Müller hat eine Problemliste nach ICPC-2 geführt und kann so die inzwischen umfangreiche Akte nach einem bestimmten Problem «filtern». Auf Wunsch von Herrn Huber überreicht ihm Dr. Müller die KG der letzten drei Jahre auf einem USB-Stick. Herr Huber wird sich in seinem neuen Ort einen Arzt suchen, der die Daten vom Stick einlesen und in seine elektronische Krankengeschichte übernehmen kann.
\end{abstract}

Im Jahr 2015 findet Herr Huber endlich diesen Hausarzt. Ein junger Kollege, der soeben seine Hausarztpraxis eröffnet hat.

Was muss erfüllt werden, damit Herr Huber allenfalls früher einen neuen Arzt findet?

Datenaustausch ergibt nur Sinn, wenn die beiden Systeme (Ursprung und Ziel) die gleichen Datencontainer zur Verfügung stellen. Es wird nie möglich sein, bis ins letzte Detail alles zu vereinheitlichen. Dies ist auch nicht nötig. Ein Eintrag zum Herzstatus sollte jedoch nach dem Transfer wieder unter Herzstatus zu finden sein und nicht unter Hautbefund. Mit den folgenden Vorschlägen wollen wir lediglich den gemeinsamen Nenner festlegen und aufzeigen, in welche Richtung die Entwicklung weitergehen soll.

Der Datenaustausch kann mit einem zu definierenden XML-Schema [2] von A nach B erfolgen.

Ein entsprechendes Mapping (d.h. Zuordnung von Dateninhalten) ermöglicht den Export vom System A in einen Transfercontainer und von dort einen Import ins System B.

\section{Label 2009 (gültig ab 1. Januar 2009)}

Schwerpunkt: Vereinheitlichung der Dokumentation, Vorbereitung Datenaustausch. 
Folgende Basiselemente der elektronischen KG müssen erfüllt sein:

\section{SOAP-Schema}

Obwohl einzelne Kollegen lieber noch weniger Struktur in einer elektronischen KG haben, darf das sogenannte SOAP-Schema [3] auch im internationalen Blickfeld als erster gemeinsamer Nenner bezeichnet werden. SOAP steht für die Begriffe Subjektiv, Objektiv, Assessment (= Beurteilung) und Prozedere. Wenn eine Krankengeschichte diese Datenfelder enthält, heisst dies, dass pro Datum oder Zeitstempel ein Eintrag nach diesem Schema in die Datenbank möglich ist. Von dort sind die Einträge jederzeit abrufbar und somit auch exportierbar.

\section{Problemliste nach ICPC-2}

Wir wollen und werden in der Schweiz den Standard etablieren, die Problem- und Diagnosenliste nach ICPC-2 [4] zu führen. Auch bei der PapierKG besteht das Problem, die Übersicht zu behalten. Dies ist mit der zunehmenden Datenmenge bei der elektronischen KG nicht anders. Wenn nun jeder sein individuelles Schema der Problemliste hat, verhindert dies den Datenaustausch und ebenso eine rasche und zuverlässige Orientierung z.B. eines Praxisassistenten, eines Praxisstellvertreters oder eben des nachfolgenden Hausarztes. Wenn es uns gelingt, die Problemliste einheitlich zu führen, werden wir einen gewaltigen Schritt vorwärtskommen auf dem Weg der Migrationsfähigkeit von medizinischen Daten.

\section{Zahlendaten in separaten Feldern erfassen} Alle beim Patienten erhobenen Zahlendaten müssen in einem separaten Feld erfasst werden.

Es ist aus Gründen der Auswertung zwingend zu wissen, wo der Blutdruckwert abgelegt wird. Blutdruckwerte, Pulswerte, Grösse, Gewicht, Bauchumfang und BMI usw. sind Daten, die als Zahlenwerte sinnvollerweise separat und nicht im Feld «Objektiv» erfasst werden, obwohl sie von der Sache her selbstverständlich zu den objektiven Daten gehören.

\section{Medikamentenmanagement}

Die Medikamente sollen auf Basis des Pharmacodes (ACT) oder analoger Systematik erfasst und die Dosierung nach dem Schema 0 - 0 - 0 - 0 miterfasst werden. Ein spezielles Feld «Unverträglichkeit von Medikamenten» muss ebenfalls zur Verfügung stehen mit dem Eintrag der Begründung.

\section{Labordaten}

Die Labordaten werden ebenfalls mit Zeitstempel und zugehörigen Referenzwerten gespeichert.
Diese Liste ist für das Label 2009 abschliessend.

Softwarelösungen, die diese Möglichkeiten anbieten, erfüllen die Anforderungen für das Label 09. Selbstverständlich sind die Firmen frei, weitere Features einzubauen, schon Anforderungen für spätere Labels zu erfüllen, grafische Auswertungen zu ermöglichen, Geräteschnittstellen usw. zu implementieren.

\section{Label 2010 (gültig ab 1. Januar 2010)}

Schwerpunkt: RFE, punktueller Datenaustausch, Uploadmöglichkeit.

Zu den Punkten 1. bis 5. von Label 2009 kommen zusätzlich:

\section{RFE}

Zusätzlich zum SOAP-Schema und zur Problemliste nach ICPC-2 besteht die Möglichkeit, den Konsultationsanlass aus Patientensicht (reason for encounter) ebenfalls nach ICPC-2 in einem separaten Feld zu erfassen. Die Software muss diese Option RFE anbieten. Der einzelne Arzt wird dies zu Beginn sehr unterschiedlich intensiv nutzen. Die Möglichkeit, den Konsultationsanlass zu erfassen, ist jedoch für hausärztliche Projekte auf verschiedenen Ebenen zwingend.

\section{Datenaustausch (KG-Transfer)}

Die Daten der Punkte 1 bis 6 können durch den User ohne Inanspruchnahme der Softwarefirma exportiert (XML-Schema) und aus einem Fremdsystem eingelesen werden.

Dadurch kann die elektronische Krankengeschichte eines Patienten von Arzt A nach Arzt B transferiert werden, und die Inhalte der Punkte 1 bis 6 sind korrekt abgelegt

\section{Upload von Daten}

Analog den Daten für die Trustcenter können klinische Daten zu Forschungszwecken anonymisiert auf einen definierten Server hochgeladen werden (XML-Schema).

Der User kann dies ohne Hilfe der Softwarefirma selbsttätig vornehmen.

Der Datensatz umfasst Daten aus den Bereichen der Punkte 2 bis 6 .

\section{Label 2011 (gültig ab 1. Januar 2011)}

Schwerpunkt: Migrationsfähigkeit, Episodenkonzept.

Das Label 2010 muss vollumfänglich erfüllt sein. Zusätzlich kommen dazu:

\section{Episodenkonzept}

Die elektronische KG ist so konzipiert, dass das der ICPC-2 zugrundeliegende Episodenkonzept [5] abgebildet wird. Dies bedeutet konkret, dass 
Langzeitverläufe, Wechsel von Problemen bei gleichbleibender Episode usw. erfasst und dargestellt werden können.

\section{Datenbankexport}

Die Datenbank ist als ganzes exportierbar. Die gespeicherten Inhalte sind exportierbar und in eine Open-Source-Datenbank (wie z.B. Postgres) importierbar. Die Migration erfolgt verlustfrei und ohne Support durch die Softwarefirma.

\section{Diskussion}

Mit der Lancierung einer Road-Map für die elektronische Krankengeschichte in der Schweiz setzen wir bewusst ein Zeichen in Richtung Datenaustausch und Migrationsfähigkeit.

Wir sind überzeugt, dass für viele Kolleginnen und Kollegen die hohen Investitionen und die fehlende Möglichkeit des Datenaustausches die Haupthindernisse sind, den Schritt zur papierarmen Praxis zu wagen. Die Möglichkeit des Austausches einer ganzen elektronischen Krankengeschichte oder Teilen davon ist längst überfällig. Langfristig gesehen darf es auch nicht sein, dass ich einem allfälligen Praxisnachfolger den Entscheid vorwegnehme, mit welchem System er einmal zu arbeiten hat.

Die Softwarefirmen sind seit 1. Januar 2008 informiert und haben Kooperationsbereitschaft signalisiert. Die SGAM.Informatics-CD-ROM «Die elektronische Krankengeschichte in der Arztpraxis» wird dieses Jahr in der dritten Auflage erscheinen. Sieben Softwarefirmen haben zugesagt, ihre Lösungen mit Screenvideos auf der $\mathrm{CD}$ zu präsentieren. Die bisherigen Drehbücher wurden durch zwei weitere Vorgaben ergänzt: interne Kommunikation/Dateimanagement und ICPC-2-Integration.

Einige Produkte erfüllen heute schon die Anforderungen des Labels 2009.

Auf der SGAM-Homepage werden per Ende 2008 diejenigen Firmen als Partner gelistet, die die Kriterien des Labels erfüllen. Die Kolleginnen und Kollegen haben somit die Möglichkeit, sich rasch zu informieren mit welcher Softwarelösung sie auch für die Zukunft gerüstet sind.

\section{Literatur}

1 SISA-Studie FMH/SGAM.Informatics Januar 2008.

2 Die «Extensible Markup Language» (engl. für «erweiterbare Auszeichnungssprache»), abgekürzt XML, ist eine Auszeichnungssprache zur Darstellung hierarchisch strukturierter Daten in Form von Textdateien. XML wird u.a. für den Austausch von Daten zwischen unterschiedlichen ITSystemen eingesetzt, speziell über das Internet. Eine Information wird durch sogenannte Tags identifiziert (z.B. $<$ BDsyst $>160<$ /BDsyst $>$ ) und kann somit eindeutig zugeordnet werden.

3 Grundlage aus: EQuiP Medical Record Project Team. Kriterien zur Beurteilung von elektronischen Krankengeschichten auf Basis der «Zusammenstellung von Qualitäts-Indikatoren zur Verbesserung der persönlichen Krankengeschichte». Zürich: compis; 2004.

4 WONCA-International Classification Committee WICC. www.globalfamilydoctor.com/wicc/.

5 Körner T, Saad A, Laux G, Rosemann T, Beyer M, Szecsenyi Joachim. Die Episode als Grundlage der Dokumentation. Dtsch Ärztebl. 2005;102(46): A 3168-72. 